\title{
Actuated Transitory Metal-Ligand Bond As Tunable Electromechanical Switch
}

\author{
Robin Ohmann, ${ }^{*}{ }^{\dagger}$ Lucia Vitali, ${ }^{\dagger,}, \S$ and Klaus Kern ${ }^{\dagger, l l}$ \\ ${ }^{\dagger}$ Max-Planck-Institut für Festkörperforschung, Heisenbergstrasse 1, D-70569, Stuttgart, Germany, ${ }^{\ddagger}$ Centro de Física \\ de Materiales CFM-MPC, Centro Mixto CSIC-UPV/EHU, E-20018, San Sebastián, Spain, ${ }^{\S}$ IKERBASQUE, Basque \\ Foundation for Science, E-48011, Bilbao, Spain, and "Institut de Physique de la Matière Condensée, Ecole Polytechnique \\ Fédérale de Lausanne (EPFL), CH-1015 Lausanne, Switzerland
}

\begin{abstract}
Electrically tunable molecules are highly attractive for the construction of molecular devices, such as switches, transistors, or machines. Here, we present a novel nanomechanical element triggered by an electrical bias as external stimulus. We demonstrate that a transitory chemical bond between a copper atom and coordinating organic molecules adsorbed on a metal surface acts as variable frequency switch, which can be actuated and probed by means of low-temperature scanning tunneling microscopy. Whereas below a threshold bias voltage the bond is permanently either formed or broken the bonding state continuously oscillates at higher voltages. The switching rate of the bistable molecular system can be widely tuned from below $1 \mathrm{~Hz}$ up to the kilohertz regime. The quantum yield per tunneling electron to trigger a transition between the two states varies spatially and is related to the local density of states of the bonded and nonbonded configuration.
\end{abstract}

KEYWORDS Conductance switching, metal-ligand bond, nanoelectromechanical systems, time-resolved spectroscopy, electron quantum yield, scanning tunneling microscopy

M anipulation of matter at the atomic scale allows unraveling the principles of naturally occurring chemical and physical processes and may open pathways for controlling and designing components for nanoscale functional devices. Recently, impressive progress has been made in modifying the position, charge state, and conformation of atoms and single molecules adsorbed on a surface by external stimuli such as light, temperature, electric fields, or tunneling electrons. ${ }^{1-18}$ For investigation of such nanoscale systems, the STM has proven to be a versatile tool as it provides a high level of spatial localization in addressing and modifying adsorbed chemical species and determining their electronic structure.

A unique characteristic of STM is the on-demand formation and breaking of chemical bonds with atomic precision. Furthermore, the electronic properties of the products can be probed locally after the process and be compared to the ones of the original components. ${ }^{5,19,20}$ Structures have typically been created by bringing the reactants close together ${ }^{20}$ and in some cases followed by applying a voltage pulse. ${ }^{21}$ Cases of nonreversible reactions have been reported $^{21}$ and recently a subsequent breaking of an artificially created bond has been shown. ${ }^{5}$ Of particular interest for molecular devices would be systems, where the external stimulus may lead either to a bond formation or a bond breaking. Metal-organic multiligand complexes might serve as ideal model systems for this purpose. The bonding

* To whom correspondence should be addressed. E-mail: r.ohmann@fkf.mpg.de. Received for review: 04/23/2010

Published on Web: 07/02/2010 strength is large enough to stabilize well-defined molecular configurations, but weak enough to make them responsive to electrical stimulus. In addition, metal-organic complexes can be engineered with atomic precision at surfaces ${ }^{22}$ and be exploited for local functionalization. ${ }^{23-25}$

Here, we demonstrate the formation of transitory metalligand bonds of a metal-organic complex triggered by inelastic electron tunneling. We used complexes formed by reacting 4-[trans-2-(pyrid-4-yl-vinyl)] benzoic acid (hereafter referred to as PVBA) with copper adatoms on the $\mathrm{Cu}$ (111) surface. $^{26,27}$ The resulting $\mathrm{Cu}(\mathrm{PVBA})_{2}$ complex has a linear structure (see scheme in Figure 1a), consisting of two PVBA molecules bonded through the pyridyl nitrogen to one central $\mathrm{Cu}$ atom. ${ }^{26}$ Measuring the tunneling current at specific positions on the complex as a function of time reveals random fluctuations between two defined states resembling a telegraph noise. We ascribe the origin of this two-level system to a transitory bond of one of the coordinating PVBA molecules to the central metal atom as a response to the applied tunneling conditions. The process can be controlled by well-defined sample bias, varying tip-sample distance (i.e., tunneling currents) and lateral position of the tip with respect to the complex.

The experiments were carried out with a home-built lowtemperature scanning tunneling microscope operated at a temperature of $6 \mathrm{~K}$ in ultrahigh vacuum. The single crystal $\mathrm{Cu}(111)$ was prepared by repeated cycles of $\mathrm{Ar}^{+}$ion sputtering and subsequent annealing. 4-[Trans-2-(pyrid-4-ylvinyl)] benzoic acid was evaporated $\left(T_{\text {cell }}=456 \mathrm{~K}\right.$ ) on the clean substrate at room temperature obtaining submono- 

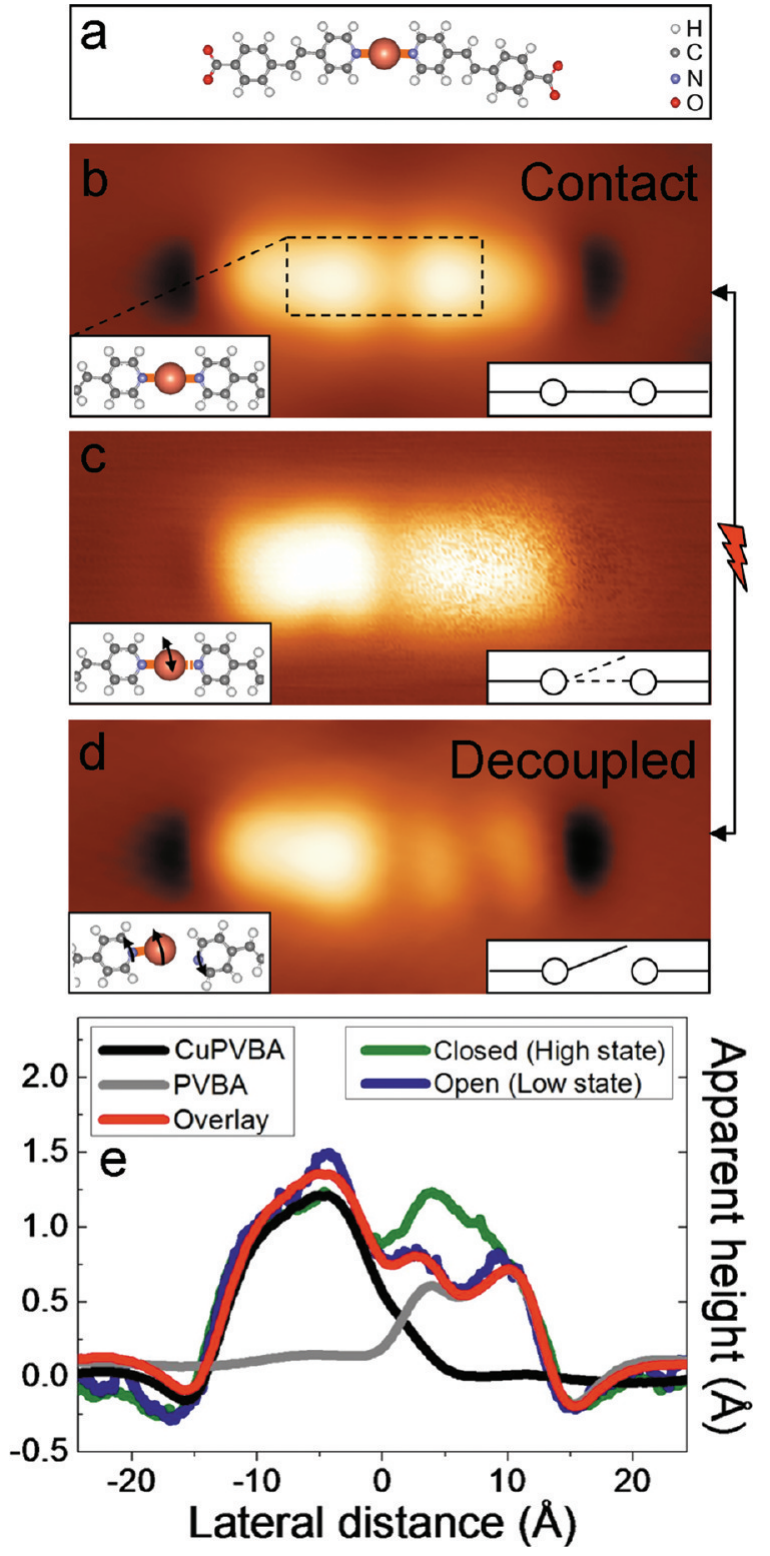

FIGURE 1. STM topography images of a self-assembled nanoswitch and schemes illustrating the mechanism. (a) Scheme of the metalorganic complex $\mathrm{Cu}(\mathrm{PVBA})_{2}$. (b) High-conductance state of the complex measured at low voltage $(-0.1 \mathrm{~V})$. (c) Same area as (b) imaged at a higher voltage $(0.8 \mathrm{~V})$. One side appears fuzzy, which is characteristic of rapid changes in the conductance. (d) Read out of the low-state at the same voltage as (b). Prior to imaging the lowstate, the complex in the high-state was brought into the low-state by applying a sufficiently high voltage on the fuzzy area (see text). In the low-state, one PVBA molecule is decoupled from the central $\mathrm{Cu}$ atom. Images are $51 \times 22 \AA^{2}$. (e) Comparison of the apparent height profiles of the high- and low-state with the profile of a single PVBA molecule and a single PVBA molecule bonded to a $\mathrm{Cu}$ adatom fabricated by single-molecule chemistry using the STM (all profiles taken at comparable voltages along the long-axis).

layer coverage. Bias voltages refer to the sample voltage with respect to the tip.

A typical STM topography image of a self-assembled $\mathrm{Cu}(\mathrm{PVBA})_{2}$ complex acquired at a sample bias of $-0.1 \mathrm{~V}$ is shown in Figure 1b. In Figure 1c we show a topograph of the same complex taken at a high bias voltage $(0.8 \mathrm{~V})$. It can be seen that one side of the metal-organic coordination compound shows a "fuzzy" appearance. This is indicative of an instability of the imaged structure triggered by tunneling electrons. ${ }^{3,12}$

To understand this behavior, the tunneling current was measured in open feedback loop conditions as a function of time. Current traces recorded in the fuzzy area show an oscillation between two values (see Figure 2a), which we label as low- and high-conductance state. The high-conductance state can be easily imaged at low voltages (see Figure $1 \mathrm{~b})$, where it is the most probable. The low-conductance state, instead, can be accessed, if the applied sample bias overcomes a threshold voltage. This causes the system to alternate between the low- and high-conductance state. A slow reduction of the voltage allows the system to relax and to restore the high-conductance state, suggesting this to be the most preferable state. However, by reducing the voltage fast, the bistable behavior can be quenched and the complex be frozen in the low-state. The system remains then stable until a sufficiently large voltage reactivates the bistable fluctuations, showing that the process is fully reversible.

As the metal complex can be either in a naturally occurring high-conductance state or have been quenched in a lowconductance state, topographic images of both states can be taken (see Figure 1b,d). Their appearance clearly indicates that the molecular configuration differs in the two cases. The apparent height profiles across the long axis in the two conductance states are shown in Figure 1e. The highconductance state (green line) shows a symmetric line profile expected for $\mathrm{Cu}(\mathrm{PVBA})_{2}$ where two PVBA molecules are coordinated to the central $\mathrm{Cu}$ atom. The profile along the low-state (blue line) differs from the previous as the apparent molecular height is reduced on the "fuzzy" side. To rationalize the profile of the low-state, we compare it with the one of a single PVBA molecule and a PVBA molecule with a single $\mathrm{Cu}$ atom coordinated to the pyridyl nitrogen (gray and black line in Figure 1e). The latter has been obtained by manipulating a $\mathrm{Cu}$ adatom toward the pyridyl end of a single PVBA molecule with the STM tip (see Supporting Information). The sum of these two line profiles (red line in Figure 1e) reproduces the one achieved on the structure in the low-conductance state. The apparent height of the low-state resembles thus an electronically decoupled PVBA monomer and an only single coordinated CuPVBA complex. These observations lead us to conclude that by applying a voltage large enough we are able to induce a transitory metal-ligand bond by attaching or detaching one PVBA molecule to or from the central $\mathrm{Cu}$ atom. As will be explained in more detail below, this process is largely governed by an asymmetric movement of the central $\mathrm{Cu}$ atom (see insets in Figure $1 \mathrm{~b}-\mathrm{d}$ ). In a broader sense, the high-state corresponds to a closed and the low-state to an open metal-organic nanocontact.

The bistable behavior of this metal-ligand bond can be controlled by several parameters. These are the tip-sample 


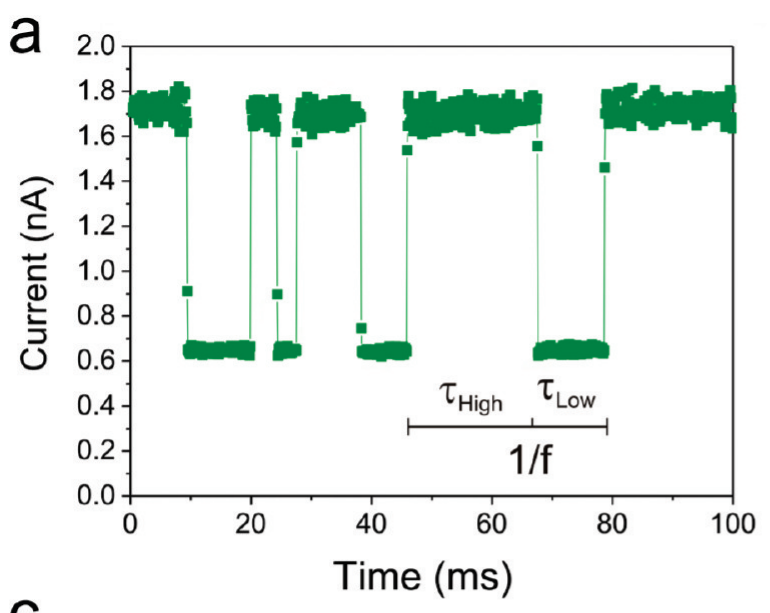

C

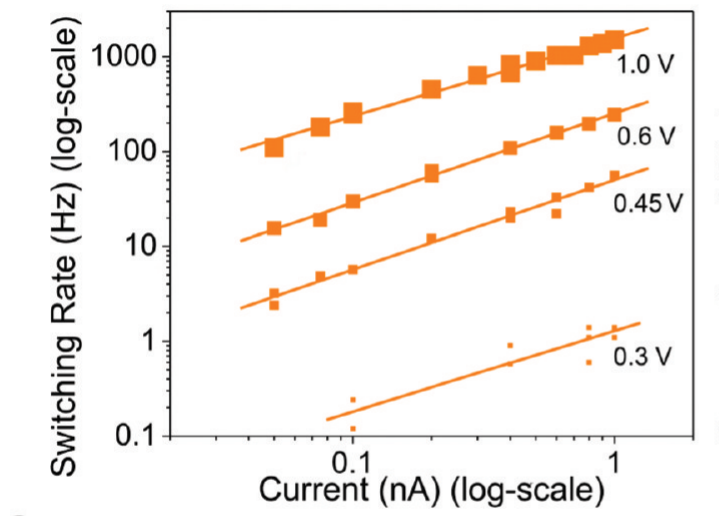

e

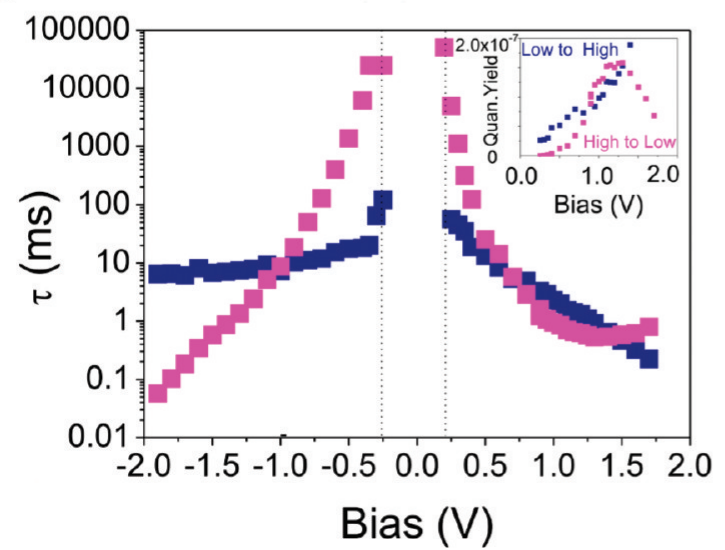

b

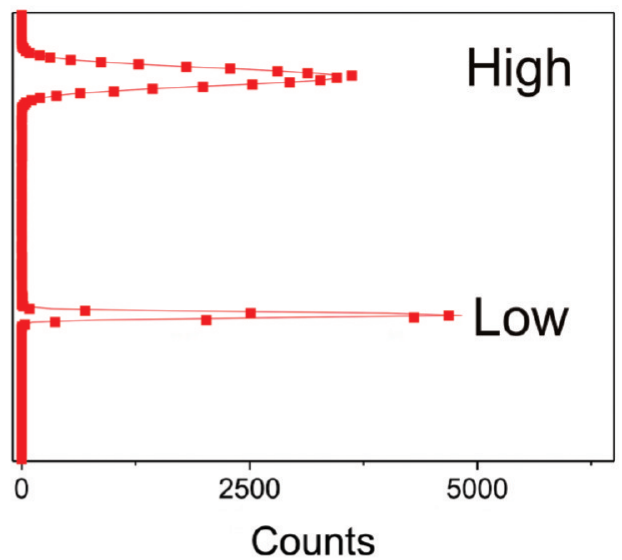

d
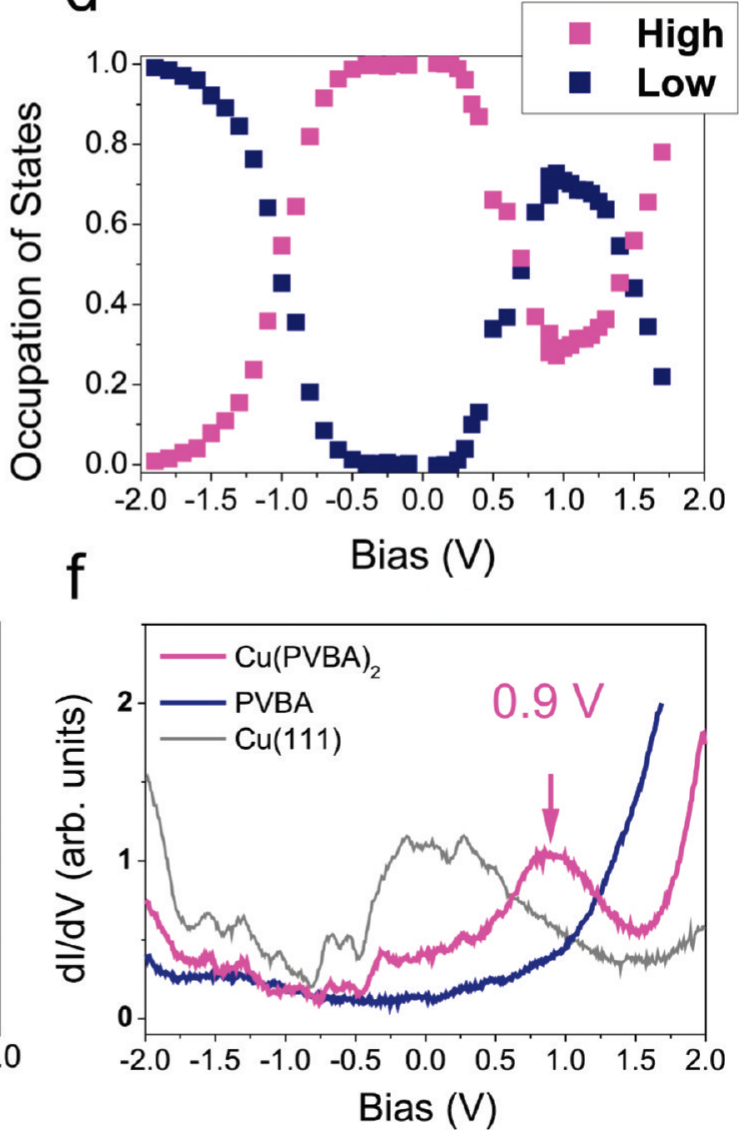

FIGURE 2. Analysis of current versus time series. (a) Current as a function of time measured in open feedback loop conditions on top of one side of the complex showing the telegraph noise. (Bias $=-1.3 \mathrm{~V}$, set point: $0.1 \mathrm{~V}, I=0.1 \mathrm{nA}$ ). (b) Corresponding histogram of the current distribution. (c) Switching rate as a function of current. (d) Probability of occupation of the low- and high-state as a function of bias voltage. (e) Lifetime of each state versus bias voltage. Inset shows the quantum yield for each transition in the positive voltage range. (f) Scanning tunneling spectra on the metal-organic complex, an isolated PVBA monomer and the bare $\mathrm{Cu}(111)$ surface for comparison.

distance (i.e., set point current), bias voltage, and position of the tip across the plane of the complex. By analyzing the resulting characteristic current fluctuations the switching rate $R$ and the lifetime $\tau_{\text {state }}$, the probability $p_{\text {state }}$ and the quantum yield of each state can be obtained (Figures 2 and 3).

The switching rate, which is the number of complete switches per second, depends linearly on the tunneling current. This is experimentally confirmed for currents up to
$10 \mathrm{nA}$ and for various positive (Figure 2c) and negative voltages (not shown). This indicates that it is a one-electron process. ${ }^{3}$ By recording the tunneling current for a sufficiently long time, histogram plots can be obtained as reported in Figure 2b. These show two characteristic peaks, whose areas represent the time spent by the complex in the high- or in the low-conductance state. In other words, the ratio of these areas, obtained from Gaussian fits, with respect to the total 

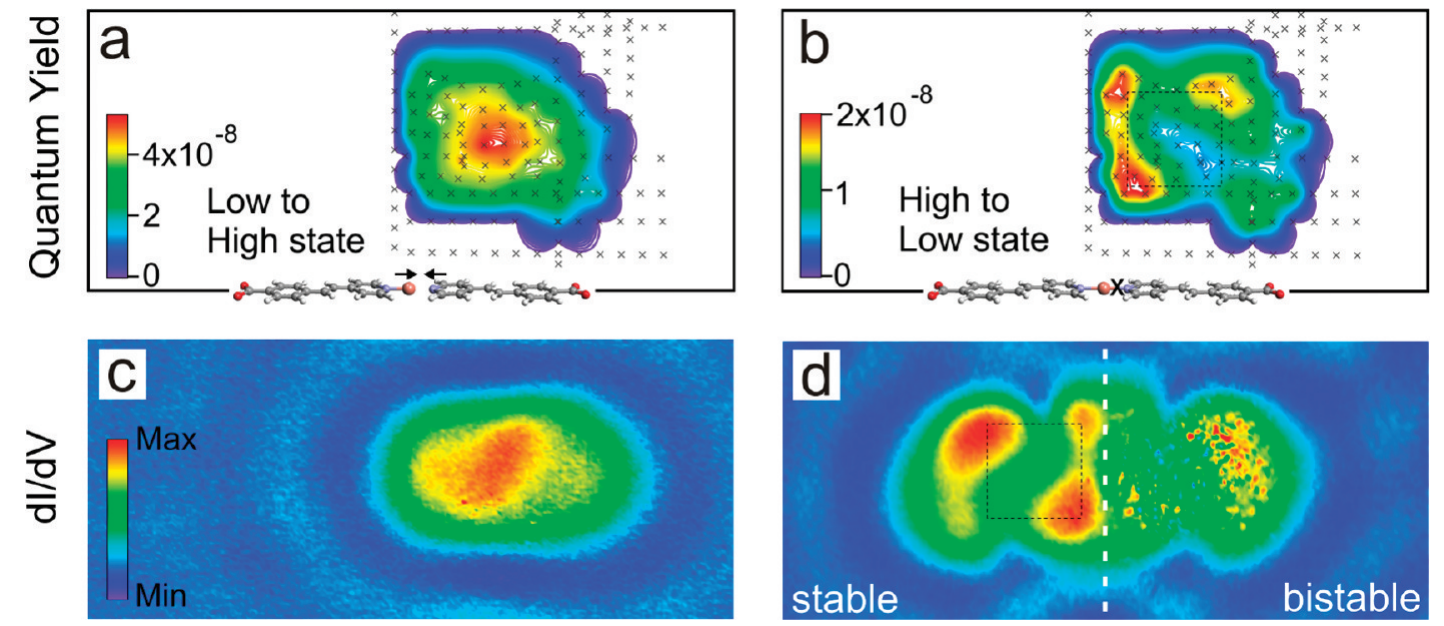

FIGURE 3. Spatial correlation of the quantum yield and the local density of states. (a,b) Contour plot of the quantum yield on the complex for the two different transitions low- to high- and high- to low-state. Red areas denote the largest quantum yield per tunneling electron. In the white area surrounding the colored area, no switching events were observed. The gray crosses indicate positions where the current traces were taken (bias $=0.9 \mathrm{~V}$, set point: $0.1 \mathrm{~V}, I=0.1 \mathrm{nA}$ ). The length of the molecules is drawn to scale. (c,d) Maps of the LDOS measured on an isolated PVBA monomer and on a $\mathrm{Cu}(\mathrm{PVBA})_{2}$ complex at the same bias voltage $(0.9 \mathrm{~V})$. Given the molecular bistability the LDOS can be better visualized at the stable side of the molecule. Measuring the LDOS at conditions of low-switching rates confirms a similar density of states at both sides of the complex. The dotted square in $(b, d)$ is a guide for the eyes for easier comparison of the features seen in the quantum yield with the LDOS. All panels are 51 by $22 \AA^{2}$.

area defines the probability $p_{\text {state }}$ to find the complex in one of the two conductance states. In Figure $2 \mathrm{~d}$, this is plotted as a function of bias voltage. It can be seen that for small voltages around the Fermi level the compound stabilizes preferably in the high-conductance state. By increasing the absolute value of the applied bias above a threshold value, the probability of the low-state increases smoothly. For negative voltages beyond $-2 \mathrm{~V}$ it approaches unity, becoming the preferred state. Acquiring a topographic image at these voltages provides then an alternative method to read out the low-state. For positive voltages, the probability of the low-state smoothly increases until it reaches a maximum around $1 \mathrm{~V}$ before it decreases again. All $\mathrm{Cu}(\mathrm{PVBA})_{2} \mathrm{com}-$ plexes measured showed the same trend.

In the following, we will relate the response of the complex to tunneling electrons to its electronic properties. A good indicator of the bistable behavior is the quantum yield (see inset Figure 2e), which is the probability of a transition (from high- to low- or low- to high-state) per tunneling electron and is given by $\operatorname{Re}\left(p_{\text {state }} I_{\text {state }}\right)$, where $e$ is the elementary charge and $I_{\text {state }}$ denotes the value of the measured current in the respective state. In Figure 3a,b, we report the quantum yield for the two transitions mapped at the voltage corresponding to the peak observed in the local density of states (LDOS) on Cu(PVBA) $)_{2}$ (Figure 2f). At each point marked by a cross, a time series was taken and evaluated in terms of the quantum yield. The resulting contour plots indicate that the quantum yield is not uniform in space and is more prominent on the molecular ligand. As a consequence, the formation of a coordination bond to the copper adatom can hence be triggered several angstroms away from the bonding site. Additionally, the spatial dependence of the quantum yield for the forward and backward reaction differs significantly. Whereas for the transition from the low- to the high-state one clear maximum (red area) is found, several maxima at well-defined positions are observed for the transition from high- to low-state. This can be understood by comparing the quantum yield with the local density of states. To measure unambiguously the density of states, a prerequisite is that the structure remains stable. This condition is met for a PVBA monomer and the other half of the PVBA complex. Their spatially resolved density of states are reported in Figure 3c,d. Since in the lowstate the PVBA molecule is electronically decoupled from the central $\mathrm{Cu}$ atom as has been shown in Figure $1 \mathrm{~d}$,e, the quantum yield for the low- to the high-state (Figure 3a) is consequently compared to the electronic structure of an isolated PVBA monomer (Figure 3c). Similar to the yield, the density of states shows one maximum. The quantum yield of the high- to the low-state (Figure 3b) is compared with the more stable half of the complex (Figure $3 \mathrm{~d}$ ), where the PVBA is bonded to the central $\mathrm{Cu}$ atom. Several maxima are observed in the conductance map, whose shape resemble the one in the yield map. Thus, both comparisons reveal clearly that the spatial dependence of the density of states matches the one of the quantum yield of each transition.

The average lifetime of each state, obtained by dividing the relative occupation of each state by the switching rate $\tau_{\text {state }}=p_{\text {state }} / R$ is shown in Figure 2e as a function of voltage. For negative voltages, the lifetime of the high-state covers a broad range extending over several orders of magnitude from below $0.1 \mathrm{~ms}$ to above $10 \mathrm{~s}$, whereas the lifetime of the low-state remains roughly constant. The dependence for positive voltages differs as the lifetime for the high-state is reaching a minimum and then increases again, reflecting the influence of the LDOS. The switching rate, which is the 

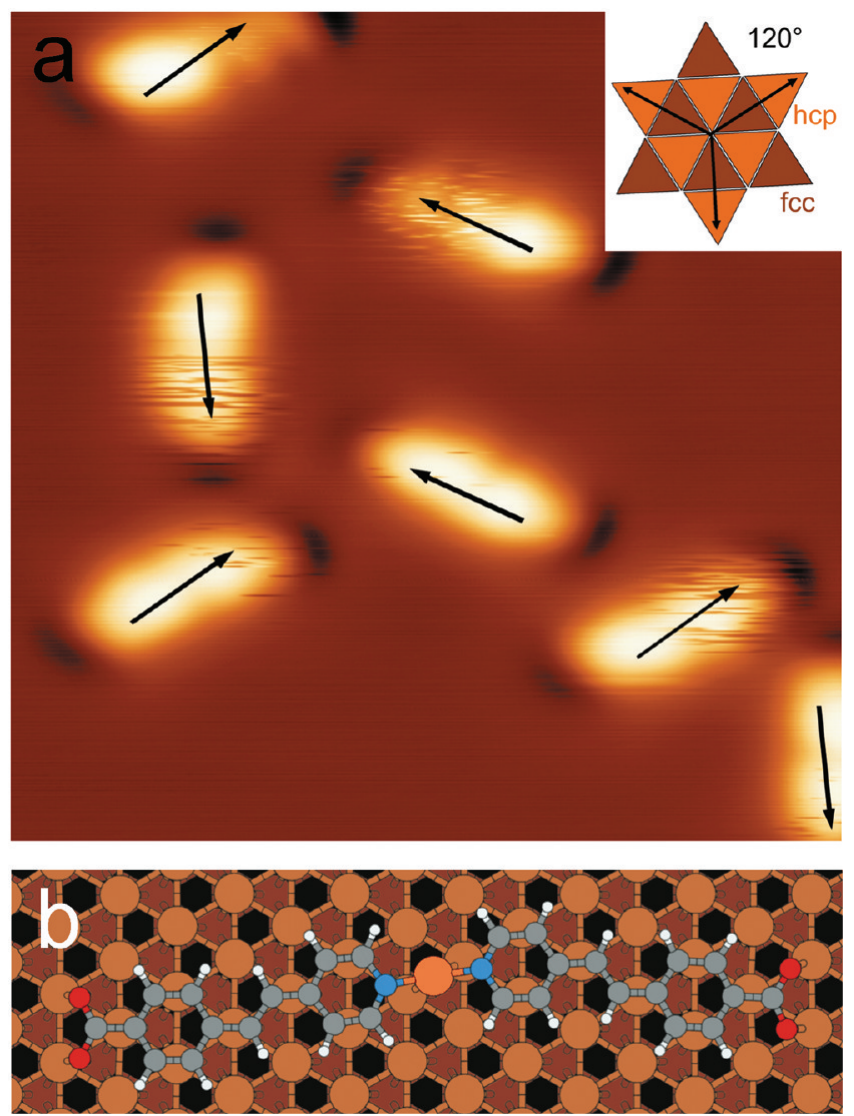

FIGURE 4. The role of the substrate in the switching mechanism. (a) Overview STM topography image showing several complexes in different orientations (bias $=-1.7 \mathrm{~V}$ ). The arrows indicate directions from the quiet side to the fuzzy side. Their distribution is 3 -fold symmetric. Image is $101 \times 101 \AA^{2}$. (b) Model of a Cu(PVBA) $)_{2}$ complex on $\mathrm{Cu}(111)$ obtained from DFT calculations ${ }^{26,28}$ showing that the second atomic layer of the substrate imposes an asymmetry to the molecular structure.

inverse of the sum of the lifetime of each state, ranges from a few events per minute to well above $1 \mathrm{kHz}$. For the higher frequencies the time resolution of the used electronics $(>20$ $\mu \mathrm{s})$ limits the measurability of shorter lifetimes and hence higher frequencies. Nevertheless, the measured switching rate in the kHz-regime shows that it is a fast process compared to other known molecular-based functional units adsorbed on a surface. ${ }^{10,13}$ The quantum yield is also appreciable; the highest measured quantum yield was in the $10^{-6}$ range. Similar values have been achieved only for an electron-induced lateral movement of a single cobalt atom attached to a Cu chain. ${ }^{3}$ Compared to molecular systems, ${ }^{7,9,29}$ this is up to 3 orders of magnitude larger.

Given the symmetry of the metal complex (Figure 1a) it may be surprising that only one of the PVBA molecules shows the described bistable behavior (see Figures 1 and 3). This asymmetry becomes evident by taking the Cu substrate into account. A large-scale STM topography image shown in Figure $4 \mathrm{a}$ illustrates that the long axis of the complex on $\mathrm{Cu}(111)$ follows well-defined crystallographic orientations. Notably, the "fuzzy" appearing half is always facing in one specific direction as indicated by the arrows, that is, we did not observe parallel aligned complexes, where in one case the "fuzzy" side is on one side and in the other case it is on the opposite side. The distribution of the complexes follows then not a 6-fold symmetry, but a 3 -fold symmetry. This suggests that the second and subsequent layer (see inset in Figure 4a) affects the bonding of the molecules at the surface and consequently the bistability of the metal complex. Figure $4 \mathrm{~b}$ reveals, indeed, that the adsorption geometry of the two halves, calculated by means of density functional theory (DFT), differs with respect to lower lying Cu layers.

The structural model also provides the basis to rationalize a possible scenario for the atomic-scale movements of the molecular switch. As has been indicated by the insets in Figure $1 \mathrm{~b}-\mathrm{d}$, the bistable behavior can be assigned to $\mathrm{a}$ movement of the $\mathrm{Cu}$ atom. Following its displacement, both PVBA ligands are slightly relaxed toward new energetically preferred positions. These are expected to be similar to the adsorption positions of one single PVBA molecule and one CuPVBA compound. ${ }^{26,28}$ The structural model resulting from DFT calculations depicted in Figure $4 \mathrm{~b}$ reveals that the $\mathrm{Cu}$ atom resides on a bridge-site for the energetically lowest configuration of the $\mathrm{Cu}(\mathrm{PVBA})_{2}$ complex. In the case of a single $\mathrm{Cu}$ adatom ${ }^{30}$ as well as for CuPVBA the hollow-site is, however, the preferred adsorption site. We thus suggest that the $\mathrm{Cu}$ atom switches between the bridge position being bonded to both ligands and a nearby hollow-site being bonded to only one of them. Whereas the actual displacements are too small to be detected, the breaking of the chemical bond can be visualized via the conductance change. Metal-ligand bonds have generally precise bond lengths differing by only a few tenth of an angstrom. ${ }^{27}$ Once the $\mathrm{Cu}$ adatom is in the hollow-site, this geometry allows the bonding to one PVBA molecule only, whereas the distance to the other molecule is too large to form a bond. If the adatom resides in the bridge position bonding can occur to both molecules. The bistability can hence be ascribed to a competition between preferred adsorption positions of the single components and the entire metal-organic complex. The switching between both configurations is steered by tunneling into electronic states of the respective chemical species.

The investigated bistable nanoscale molecular system offers interesting prospects for designing large-scale assemblies of functional units. It provides a highly localized switch with a tunable rate up to the kilohertz regime. Furthermore, nanoelectromechanical devices could be envisioned based on injecting electrons with defined energies into bistable configurations triggering an opening or closing of a nanocontact.

Acknowledgment. We thank Giacomo Levita for sharing his results prior to publication and Mario Ruben for synthesizing the PVBA.

Supporting Information Available. Illustration of the synthesis of a CuPVBA complex with low-temperature scan- 
ning tunneling microscopy. This material is available free of charge via the Internet at http://pubs.acs.org.

\section{REFERENCES AND NOTES}

(1) Balzani, V.; Credi, A.; Venturi, M. Molecular Devices and Machines Wiley-VCH: Weinheim, 2008.

(2) Eigler, D. M.; Lutz, C. P.; Rudge, W. E. Nature 1991, 352, 600

(3) Stroscio, J. A.; Tavazza, F.; Crain, J. N.; Celotta, R. J.; Chaka, A. M Science 2006, 313, 948

(4) Stipe, B. C.; Rezaei, M. A.; Ho, W. Science 1998, 279, 1907

(5) Repp, J.; Meyer, G.; Paavilainen, S.; Olsson, F. E.; Persson, M. Science 2006, 312, 1196.

(6) Gaudioso, J.; Ho, W. Angew. Chem., Int. Ed. 2001, 40, 4080.

(7) Lastapis, M.; Martin, M.; Riedel, D.; Hellner, L.; Comtet, G.; Dujardin, G. Science 2005, 308, 1000.

(8) Harikumar, K. R.; Polanyi, J. C.; Sloan, P. A.; Ayissi, S.; Hofer, W. A.J. Am. Chem. Soc. 2006, 128, 16791

(9) Iancu, V.; Hla, S.-W. Proc. Natl. Acad. Sci. U.S.A. 2006, 103, 13718

(10) Liljeroth, P.; Repp, J.; Meyer, G. Science 2007, 317, 1203.

(11) Katano, S.; Kim, Y.; Hori, M.; Trenary, M.; Kawai, M. Science 2007, 316, 1883.

(12) Henningsen, N.; Franke, K. J.; Torrente, I. F.; Schulze, G.; Priewisch, B.; Rück-Braun, K.; Dokić, J.; Klamroth, T.; Saalfrank, P.; Pascual, J. I. J. Phys. Chem. C 2007, 111, 14843

(13) Wang, Y.; Kröger, J.; Berndt, R.; Hofer, W. A. J. Am. Chem. Soc. 2009, 131, 3639

(14) Repp, J.; Meyer, G.; Olsson, F. E.; Persson, M. Science 2004, 305 493.

(15) Wu, S. W.; Ogawa, N.; Ho, W. Science 2006, 312, 1362
(16) Comstock, M. J.; Levy, N.; Kirakosian, A.; Cho, J.; Lauterwasser, F.; Harvey, J. H.; Strubbe, D. A.; Fréchet, J. M. J.; Trauner, D.; Louie, S. G.; Crommie, M. F. Phys. Rev. Lett. 2007, 99, No. 038301

(17) Alemani, M.; Peters, M. V.; Hecht, S.; Rieder, K.-H.; Moresco, F.; Grill, L. J. Am. Chem. Soc. 2006, 128, 14446.

(18) Henzl, J.; Mehlhorn, M.; Gawronski, H.; Rieder, K.-H.; Morgenstern, K. Angew. Chem., Int. Ed. 2006, 45, 603.

(19) Lee, H. J.; Ho, W. Science 1999, 286, 1719

(20) Nazin, G. V.; Qiu, X. H.; Ho, W. Science 2003, 302, 77

(21) Hla, S.-W.; Bartels, L.; Meyer, G.; Rieder, K.-H. Phys. Rev. Lett. 2000, 85, 2777.

(22) Lingenfelder, M.; Spillmann, H.; Dmitriev, A.; Stepanow, S.; Lin, N.; Barth, J. V.; Kern, K. Chem.-Eur.J. 2004, 10, 1913.

(23) Barth, J. V.; Costantini, G.; Kern, K. Nature 2005, 437, 671.

(24) Stepanow, S.; Lingenfelder, M.; Dmitriev, A.; Spillmann, H.; Delvigne, E.; Lin, N.; Deng, X.; Cai, C.; Barth, J. V.; Kern, K. Nat. Mater. 2004, 3, 229.

(25) Gambardella, P.; Stepanow, S.; Dmitriev, A.; Honolka, J.; Groot, F. M. F. de; Lingenfelder, M.; Sen Gupta, S.; Sarma, D. D.; Bencok, P.; Stanescu, S.; Clair, S.; Pons, S.; Lin, N.; Seitsonen, A. P.; Brune, H.; Barth, J. V.; Kern, K. Nat. Mater. 2009, 8, 189.

(26) Vitali, L.; Levita, G.; Ohmann, R.; Comisso, A.; Vita, A. De; Kern, K. Nat. Mater. 2010,9,320.

(27) Tait, S. L.; Langner, A.; Lin, N.; Stepanow, S.; Rajadurai, C.; Ruben, M.; Kern, K. J. Phys. Chem. C 2007, 111, 10982.

(28) Ohmann, R.; Levita, G.; Vitali, L.; Vita, A. De; Kern K. unpublished.

(29) Wang, Y.; Ge, X.; Schull, G.; Berndt, R.; Tang, H.; Bornholdt, C.; Koehler, F.; Herges, R. J. Am. Chem. Soc. 2010, 132, 1196.

(30) Repp, J.; Meyer, G.; Rieder, K.-H.; Hyldgaard, P. Phys. Rev. Lett. 2003, 91, 206102 